\title{
Hybrid PSO-SA algorithm for training a Neural Network for Classification
}

\author{
Sriram G. Sanjeevi ${ }^{1}$, A. Naga Nikhila ${ }^{2}$,Thaseem Khan $^{3}$ and G. Sumathi ${ }^{4}$ \\ ${ }^{1}$ Associate Professor, Dept. of CSE, National Institute of Technology, Warangal, A.P., \\ India \\ sgsenitw.ac.in \\ ${ }^{2}$ Dept. of Comp. Science \& Engg., National Institute of Technology, Warangal, A.P., \\ India \\ 108nikhila.an@gmail.com \\ ${ }^{3}$ Dept. of Comp. Science \& Engg., National Institute of Technology, Warangal, A.P., \\ India \\ thaseem7@gmail.com \\ ${ }^{4}$ Dept. of Comp. Science \& Engg., National Institute of Technology, Warangal, A.P., \\ India \\ sumathiguguloth@gmail. com
}

\begin{abstract}
In this work, we propose a Hybrid particle swarm optimization-Simulated annealing algorithm and present a comparison with i) Simulated annealing algorithm and ii) Back propagation algorithm for training neural networks. These neural networks were then tested on a classification task. In particle swarm optimization behaviour of a particle is influenced by the experiential knowledge of the particle as well as socially exchanged information. Particle swarm optimization follows a parallel search strategy. In simulated annealing uphill moves are made in the search space in a stochastic fashion in addition to the downhill moves. Simulated annealing therefore has better scope of escaping local minima and reach a global minimum in the search space. Thus simulated annealing gives a selective randomness to the search. Back propagation algorithm uses gradient descent approach search for minimizing the error. Our goal of global minima in the task being done here is to come to lowest energy state, where energy state is being modelled as the sum of the squares of the error between the target and observed output values for all the training samples. We compared the performance of the neural networks of identical architectures trained by the i) Hybrid particle swarm optimization-simulated annealing, ii) Simulated annealing and iii) Back propagation algorithms respectively on a classification task and noted the results obtained. Neural network trained by Hybrid particle swarm optimization-simulated annealing has given better results compared to the neural networks trained by the Simulated annealing and Back propagation algorithms in the tests conducted by us.
\end{abstract}

\section{KEYWORDS}

Classification, Hybrid particle swarm optimization-Simulated annealing, Simulated Annealing, Gradient Descent Search, Neural Network etc.

\section{INTRODUCTION}

Classification is an important activity of machine learning. Various algorithms are conventionally used for classification task namely, Decision tree learning using ID3[1], Concept learning using 
Candidate elimination [2], Neural networks [3], Naïve Bayes classifier [4] are some of the traditional methods used for classification. Ever since back propagation algorithm was invented and popularized by [3], [5] and [6] neural networks were actively used for classification. However, since back-propagation method follows hill climbing approach, it is susceptible to occurrence of local minima. We examine the use of i) Hybrid particle swarm optimizationsimulated annealing ii) simulated annealing and iii) backpropagation algorithms to train the neural networks. We study and compare the performance of the neural networks trained by these three algorithms on a classification task.

\section{Architecture of Neural Network}

Neural network designed for the classification task has the following architecture. It has four input units, three hidden units and three output units in the input layer, hidden layer and output layer respectively. Sigmoid activation functions were used with hidden and output units. Figure 1 shows the architectural diagram of the neural network. Neurons are connected in the feed forward fashion as shown. Neural Network has 12 weights between input and hidden layer and 9 weights between hidden and output layer. So the total number of weights in the network are 21 .

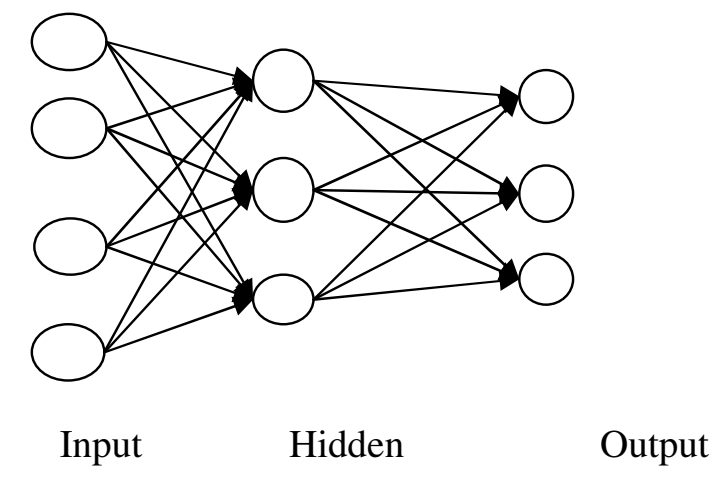

Fig. 1 Architecture of Neural Network used

\subsection{Iris data}

Neural Network shown in figure 1 is used to perform classification task on IRIS data. The data was taken from the Univ. of California, Irvine (UCI), Machine learning repository. Iris data consists of 150 input-output vector pairs. Each input vector consists of a 4 tuple having four attribute values corresponding to the four input attributes respectively. Based on the input vector, output vector gives class to which it belongs. Each output vector is a 3 tuple and will have a ' 1 ' in first, second or third positions and zeros in rest two positions, thereby indicating the class to which the input vector being considered belongs. Hence, we use 1-of- $n$ encoding on the output side for denoting the class value. The data of 150 input-output pairs is divided randomly into two parts to create the training set and test set respectively. Data from training set is used to train the neural network and data from the test set is used for test purposes. Few samples of IRIS data are shown in table 1.

\section{Simulated AnNealing to Train the NeUral NeTWORK}

Simulated annealing [7], [8] was originally proposed as a process which mimics the physical process of annealing wherein molten metal is cooled gradually from high energy state attained at high temperatures to low temperature by following an annealing schedule. Annealing schedule defines how the temperature is gradually decreased. The goal is to make the metal reach a minimum attainable energy state. In the simulated annealing lower energy states are produced in 
succeeding transitions. However, a higher energy state is also allowed to be a successor state from a given current state with a certain probability. Hence, movement to higher energy states is also allowed. The probability of these possible uphill moves decreases as the temperature is decreased.

Table 1. Sample of IRIS data used.

\begin{tabular}{|c|c|c|c|c|c|c|c|}
\hline S. No. & Attr. 1 & Attr. 2 & Attr. 3 & Attr. 4 & Class 1 & Class 2 & Class 3 \\
\hline 1 & 0.224 & 0.624 & 0.067 & 0.043 & 1 & 0 & 0 \\
\hline 2 & 0.749 & 0.502 & 0.627 & 0.541 & 0 & 1 & 0 \\
\hline 3 & 0.557 & 0.541 & 0.847 & 1 & 0 & 0 & 1 \\
\hline 4 & 0.11 & 0.502 & 0.051 & 0.043 & 1 & 0 & 0 \\
\hline 5 & 0.722 & 0.459 & 0.663 & 0.584 & 0 & 1 & 0 \\
\hline
\end{tabular}

While error back propagation algorithm follows the concept of hill climbing, Simulated annealing allows some down hill movements to be made stochastically, in addition to making the uphill moves. In the context of a neural network, we actually need to minimize our objective function. Simulated annealing tries to produce the minimal energy state. It mimics the physical annealing process wherein molten metal at high temperatures is cooled according to an annealing schedule and brought down to a low temperature with minimal energy. Simulated annealing can be used to solve an optimization problem. The objective function required to be minimized for training the neural network is the error function $E$.

We define the error function $\mathrm{E}$ as follows. The error function $\mathrm{E}$ is given by

$$
E(\vec{w}) \equiv \frac{1}{2} \sum_{x \in X} \sum_{k \in \text { outputs }}\left(t_{k x}-a_{k x}\right)^{2}
$$

where $x$ is a specific training example and $X$ is the set of all training examples, $t_{\bar{R} x}$ denotes the target output for the $k_{t h}$ output neuron corresponding to training sample $x$, $a_{h x}$ denotes the observed output for the $k_{t h}$ output neuron corresponding to training sample $x$. Hence, error function computed is a measure of the sum of the squares of the error between target and observed output values for all the output neurons, across all the training samples.

\subsection{Simulated Annealing Algorithm}

Here we describe the Simulated Annealing algorithm used for training the neural network.

1.Initialize the weights of the neural network shown in figure 1 to small random values.

2.Evaluate the objective function $E$ shown in equation (1) for the neural network with the present configuration of weights used in the neural network.

Follow a temperature annealing schedule with the algorithm.

3.Change one randomly selected weight of the neural network with a random perturbation. Obtain the objective function $E$ for the neural network with the changed configuration of weights. Also compute $\Delta E=(E$ value for the previous network before weight change $)$ - $(E$ value for the present network with changed configuration of weights). 
4.If $\Delta E$ is positive then new value of $E$ is smaller and therefore better than previous value. Then accept the new state and make it the current state. else accept the new state (even though it has a higher $E$ value) with a probability $p$ defined by

$$
p=e^{-\Delta E / T}
$$

5.Revise the temperature $T$ as per schedule defined.

6.Go to step 3 if $E$ value obtained is not yet satisfactory else return solution. Solution is the present configuration of the weights in the neural network.

\subsection{Encoding of the Weights in the Neural Network}

The Neural Network in figure 1 has 12 weights between input and hidden layer and 9 weights between hidden and output layer. So the total number of weights in the network are 21 . We coded these weights in binary form. Each weight was assumed to vary between +12.75 and -12.75 with a precision of 0.05 . This is because weights learned by a typical neural network will be small positive or negative real numbers. Any real number between -12.75 and +12.75 with a precision of 0.05 can be represented by a 9-bit binary string. Thus, -12.75 was represented with a binary string '000000000' and +12.75 was represented with '111111111'. For example, number 0.1 is represented by '100000010'. Each of the twenty one weights present in the neural network was represented by a separate 9 bit binary string and the set of all the weights present in the neural network is represented by their concatenation having 189 bits. These 189 bits represent one possible assignment of values to the weights in the neural network.

Initially, a random binary string having 189 bits was generated to make the assignment of weights randomly for the network. We obtain the value of objective function $E$ for this assignment of weights to the neural network. Simulated Annealing requires a random perturbation to be given to the network. We do this by selecting randomly one of the 189 binary bits and flipping it. If the selected bit was 1 it is changed to 0 and if it was $O$ it is changed to 1 . For the new configuration of network with weight change objective function $E$ is calculated. Then, $\Delta E$ was calculated as ( $E$ value for the previous network before weight change $)$ - $E$ value for the present network with changed configuration of weights). Using this approach, simulated annealing algorithm shown in section 3.1 was executed following a temperature schedule.

The temperature schedule followed is defined as follows:

The starting temperature is taken as $T=1050$;

Current temp $=T / \log ($ iterations +1$)$;

Current temperature was changed after every 100 iterations using above formula.

\section{ERror BACK-Propagation}

Error-Back propagation algorithm uses gradient descent search. Gradient descent approach searches for the weight vector that minimizes error $E$. Towards this, it starts from an arbitrary initial weight vector and then changes it in small steps. At each of these steps the weight is altered in the direction which produces steepest descent along the error surface. Hence, gradient descent approach follows the hill climbing approach.

The direction of the steepest descent along the error surface is found by computing the derivative of $E$ with respect to the weight vector $\mathbf{w}$.

Gradient of $E$ with respect to $w$ is $=\nabla E(w) \equiv\left[\frac{\partial E}{\partial w_{a}}, \frac{\partial E}{\partial w_{2}}, \ldots, \frac{\partial E}{\partial w_{n}}\right]$ 
International Journal of Computer Science, Engineering and Applications (IJCSEA) Vol.1, No.6, December 2011

\subsection{Algorithm for Back-propagation}

Since the back propagation algorithm has been introduced [3], [5] it has been used widely as the training algorithm for training the weights of the feed forward neural network. It was able to overcome the limitation faced by the perceptron since it can be used for classification for nonlinearly separable problems unlike perceptron. Perceptron can only solve linearly separable problems. Main disadvantage of neural network using back propagation algorithm is that since it uses hill climbing approach it can get stuck at local minima. Below we present in table 2 the back propagation algorithm [9] for the feed forward neural network. It describes the Back propagation algorithm using batch update wherein weight updates are computed for each weight in the network after considering all the training samples in a single iteration or epoch. It takes many iterations or epochs to complete the training of the neural network.

Table 2. Back propagation Algorithm

\section{Back propagation algorithm}

Each training sample is a pair of the form $(\overrightarrow{\boldsymbol{x}}, \overrightarrow{\boldsymbol{t}})$, where $\boldsymbol{x}$ is the input vector, and $\boldsymbol{t}$ is the target vector.

$\mu$ is the learning rate, $m$ is the number of network inputs to the neural network, $y$ is the number of units in the hidden layer, $z$ is the number of output units.

The input from unit $i$ into unit $j$ is denoted by $x_{j i}$, and the weight from unit $i$ into unit $j$ is denoted by $w_{j i}$.

- Create a feed-forward network with $m$ inputs, $y$ hidden units, and $z$ output units.

- Initialize all network weights to small random numbers.

- Until the termination condition is satisfied, do

- For each $\langle\overrightarrow{\boldsymbol{x}}, \overrightarrow{\boldsymbol{t}}\rangle$ in training samples, do

Propagate the input vector forward through the network:

1. Input the instance $x$ to the network and compute the output $\mathrm{o}_{\mathrm{u}}$ of every unit $u$ in the network.

Propagate the errors backward through the network:

2. For each network output unit $k$, with target output $t_{k_{k}}$ calculate its error term $\delta_{\hat{k i}}$

$\sigma_{k}-o_{k}\left(1-o_{k}\right)\left(t_{k}-o_{k}\right)$

3. For each hidden unit $h$, calculate its error term $\delta_{\text {h }}$

$$
\delta_{h}-o_{h}\left(1-o_{h}\right) \sum_{k=\alpha m p z} w_{k h} \delta_{k}
$$

4. For each network weight $w_{j i}$, do

$$
\Delta u_{\mathrm{ji}}-\Delta w_{j \mathrm{i}}+\eta \delta_{j} x_{\mathrm{ji}}
$$

- For each network weight $w_{j i}$, do

$$
w_{j l}-w_{j l}+\Delta w_{j l}
$$




\section{HYBRID PSO-SA ALGORITHM}

In particle swarm optimization [11, 12] a swarm of particles are flown through a multidimensional search space. Position of each particle represents a potential solution. Position of each particle is changed by adding a velocity vector to it. Velocity vector is influenced by the experiential knowledge of the particle as well as socially exchanged information. The experiential knowledge of a particle $A$ describes the distance of the particle $A$ from its own best position since the particle $A$ 's first time step. This best position of a particle is referred to as the personal best of the particle. The global best position in a swarm at a time $t$ is the best position found in the swarm of particles at the time $t$. The socially exchanged information of a particle $A$ describes the distance of a particle $A$ from the global best position in the swarm at time $t$. The experiential knowledge and socially exchanged information are also referred to as cognitive and social components respectively. We propose here the hybrid PSO-SA algorithm in table 3.

Table 3. Hybrid Particle swarm optimization-Simulated annealing algorithm

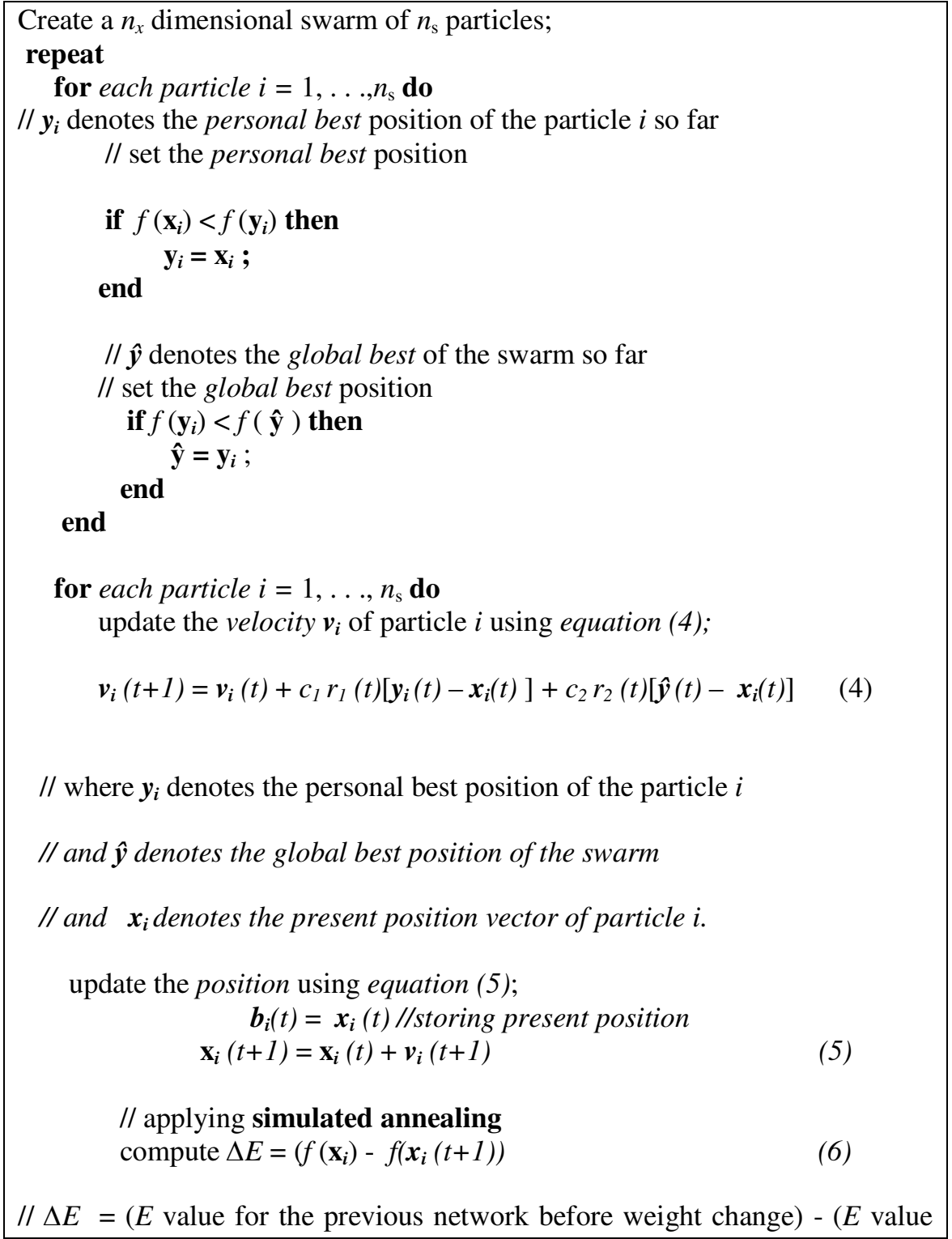

// where $y_{i}$ denotes the personal best position of the particle $i$

// and $\hat{\boldsymbol{y}}$ denotes the global best position of the swarm

// and $\boldsymbol{x}_{\boldsymbol{i}}$ denotes the present position vector of particle $i$.

update the position using equation (5);

$$
\begin{aligned}
\quad b_{i}(t)=\boldsymbol{x}_{\boldsymbol{i}}(t) / / \text { storing present position } \\
\mathbf{x}_{\boldsymbol{i}}(t+1)=\mathbf{x}_{\boldsymbol{i}}(t)+\boldsymbol{v}_{\boldsymbol{i}}(t+1)
\end{aligned}
$$

// applying simulated annealing compute $\Delta E=\left(f\left(\mathbf{x}_{i}\right)-f\left(\boldsymbol{x}_{i}(t+1)\right)\right.$

$/ / \Delta E=(E$ value for the previous network before weight change $)-(E$ value 
International Journal of Computer Science, Engineering and Applications (IJCSEA) Vol.1, No.6, December 2011

for the present network with changed configuration of weights).

- if $\Delta E$ is positive then

$/ /$ new value of $E$ is smaller and therefore better than previous value. Then

// accept the new position and make it the current position

else accept the new position $\boldsymbol{x}_{\boldsymbol{i}}(t+1)$ (even though it has a higher $E$ value) with a probability $p$ defined by

$$
p=e^{-i E / T}
$$

- Revise the temperature $T$ as per schedule defined below.

The temperature schedule followed is defined as follows:

The starting temperature is taken as $T=1050$;

Current temp $=T / \log$ (iterations +1$)$;

Current temperature was changed after every 100 iterations using above formula.

end

until stopping condition is true;

In equation (4) of table $3, \boldsymbol{v}_{\boldsymbol{i}}, \boldsymbol{y}_{\boldsymbol{i}}$ and $\hat{\boldsymbol{y}}$ are vectors of $n_{x}$ dimensions. $v_{i j}$ denotes scalar component of $\boldsymbol{v}_{i}$ in dimension $j . v_{i j}$ is calculated as shown in equation 7 where $r_{1 j}$ and $r_{2 j}$ are random values in the range $[0,1]$ and $c 1$ and $c 2$ are learning factors chosen as $c 1=c 2=2$.

$$
\left.v_{i j}(t+1)=v_{i j}(t)+c_{1} r_{l j}(t)\left[y_{i j}(t)-x_{i j}(t)\right]+c_{2} r_{2 j}(t)\left[\hat{y}_{j}(t)-x_{i j}\right)(t)\right]
$$

\subsection{Implementation details of Hybrid PSO-SA algorithm for training a neural network}

We describe here the implementation details of hybrid PSO-SA algorithm for training the neural network. There are 21 weights in the neural network shown in figure 1.

Hybrid PSO-SA algorithm combines particle swarm optimization algorithm with simulated annealing approach in the context of training a neural network. The swarm is initialized with a population of 50 particles. Each particle has 21 weights. Each weight value corresponds to a position in a particular dimension of the particle. Since there are 21 weights for each particle, there are therefore 21 dimensions. Hence, position vector of each particle corresponds to a 21 dimensional weight vector. Position (weight) in each dimension is modified by adding velocity value to it in that dimension.

Each particle's velocity vector is updated by considering the personal best position of the particle, global best position of the entire swarm and the present position vector of the particle as shown in equation (4) of table 3 . Velocity of a particle $i$ in dimension $j$ is calculated as shown in equation (8).

Hybrid PSO-SA algorithm combines pso algorithm with simulated annealing approach. Each of the 50 particles in the swarm is associated with 21 weights present in the neural network. The error function $E(w)$ which is a function of the weights in the neural network as defined in equation (1) is treated as the fitness function. Error $E(\boldsymbol{w})$ ( fitness function) needs to be minimized. For each of the 50 particles in the swarm, the solution (position) given by the 
particle is accepted if the change in error function $\Delta E$ as defined in equation 6 is positive, since this indicates error function is reducing in the present iteration compared to the previous iteration value. If $\Delta E$ is not positive then new position is accepted with a probability $p$ given by formula in equation (7). This is implemented by generating a random number between 0 and 1 . If the number generated is lesser than $p$ then the new position is accepted, else the previous position of particle is retained without changing. Each particle's personal best position and the global best position of the swarm are updated after each iteration. Hybrid PSO-SA algorithm was run for 15000 iterations. After 15000 iterations global best position gbest of the swarm is returned as the solution. Hence, stopping criterion for the algorithm was chosen as completion of 15000 iterations.

Hybrid PSO-SA algorithm combines parallel search approach of $P S O$ and selective random search and global search properties of simulated annealing and hence combines the advantages of both the approaches.

\section{EXPERIMENTS AND RESULTS}

We have trained the neural network with architecture shown in figure 1 with back propagation algorithm on training set taken from the IRIS data. Training set was a subset of samples chosen randomly from the IRIS data. Remaining samples from IRIS data were included in the test set. Performance was observed on the test data set for predicting the class of each sample.

Table 4. Results of testing for Neural Network using Back propagation

\begin{tabular}{|c|c|c|c|c|}
\hline Sl. No & $\begin{array}{c}\text { Samples in } \\
\text { Training Set }\end{array}$ & $\begin{array}{c}\text { Samples in } \\
\text { Test Set }\end{array}$ & $\begin{array}{c}\text { Correct } \\
\text { classifications }\end{array}$ & Misclassifications \\
\hline 1 & 100 & 50 & 40 & 10 \\
\hline 2 & 95 & 55 & 37 & 18 \\
\hline 3 & 85 & 65 & 42 & 26 \\
\hline 4 & 75 & 75 & 49 & \\
\hline
\end{tabular}

We have chosen similarly, training sets of varying sizes from the IRIS data and included each time the samples which were not selected for training set into test set. Neural network was trained by each of the training sets and tested the performance of the network on corresponding test sets. Training was done for 15000 iterations with each of the training sets. The value of learning rate $\mu$ used was 0.2 . Results are shown in table 4 . 
International Journal of Computer Science, Engineering and Applications (IJCSEA) Vol.1, No.6, December 2011

Table 5. Results of testing for Neural Network using Simulated annealing algorithm

\begin{tabular}{|c|c|c|c|c|}
\hline Sl. No & $\begin{array}{c}\text { Samples in } \\
\text { Training Set }\end{array}$ & $\begin{array}{c}\text { Samples in } \\
\text { Test Set }\end{array}$ & Correct classifications & Misclassifications \\
\hline 1 & 100 & 50 & 44 & 6 \\
\hline 2 & 95 & 55 & 40 & 15 \\
\hline 3 & 85 & 65 & 50 & 15 \\
\hline 4 & 75 & 75 & 59 & 16 \\
\hline
\end{tabular}

We have also trained the neural network with architecture shown in figure 1 with simulated annealing algorithm described in section 3 , with each of the training sets chosen above in table 4 and tested the performance of the network on corresponding test sets. Training was done using simulated annealing algorithm for 15000 iterations with each of the training sets. The results are shown in table 5.

We have also trained the neural network with architecture shown in figure 1 with Hybrid PSO-SA algorithm described in section 5, with each of the training sets chosen above in table 4 and tested the performance of the network on corresponding test sets. Training was done using Hybrid PSOSA algorithm for 15000 iterations with each of the training sets. The results are shown in table 6.

Table 6. Results of testing for Neural Network using Hybrid PSO-SA algorithm

\begin{tabular}{|c|c|c|c|c|}
\hline Sl.No & $\begin{array}{c}\text { Samples in } \\
\text { Training Set }\end{array}$ & $\begin{array}{c}\text { Samples in } \\
\text { Test Set }\end{array}$ & $\begin{array}{c}\text { Correct } \\
\text { Classifications }\end{array}$ & Misclassifications \\
\hline 1 & 100 & 50 & 47 & 3 \\
\hline 2 & 95 & 55 & 51 & 4 \\
\hline 3 & 85 & 65 & 61 & 5 \\
\hline 4 & 75 & 75 & 70 & 4 \\
\hline
\end{tabular}

Three neural networks with same architecture as shown in figure 1 were used for training and testing with the three algorithms of Hybrid PSO-SA algorithm, simulated annealing algorithm and back propagation algorithm respectively. Training was performed for same number of 15000 iterations on each neural network. Same training and test sets were used for comparison of neural networks performance with all the three algorithms. Results of experiments and testing point out that neural network trained with Hybrid PSO-SA algorithm gives better performance over neural networks trained with the simulated annealing and back propagation algorithms respectively across the training and test sets used. 
International Journal of Computer Science, Engineering and Applications (IJCSEA) Vol.1, No.6, December 2011

\section{Conclusions}

Our objective was to compare the performance of feed-forward neural network trained with Hybrid PSO-SA algorithm with the neural networks trained by simulated annealing and back propagation algorithm respectively. The task we have tested using neural networks trained separately using these three algorithms is the IRIS data classification. We found that neural network trained with Hybrid PSO-SA algorithm has given better classification performance among the three. Neural network trained with Hybrid PSO-SA algorithm combines parallel search approach of $P S O$ and selective random search and global search properties of simulated annealing and hence combines the advantages of both the approaches. Hybrid PSO-SA algorithm has performed better than simulated annealing algorithm using its parallel search strategy with a swarm of particles. It was able to avoid the local minima by stochastically accepting uphill moves in addition to the making the normal downhill moves across the error surface. It has given better classification performance over neural network trained by back-propagation. In general, neural networks trained by back propagation algorithm are susceptible for falling in local minima. Hence, Hybrid PSO-SA algorithm has given better results for training a neural network and is a better alternative to both the Simulated annealing and Back propagation algorithms.

\section{REFERENCES}

[1] Quinlan, J.R.(1986). Induction of decision trees. Machine Learning, 1 (1), 81-106.

[2] Mitchell, T.M.(1977). Version spaces: A candidate elimination approach to rule learning. Fifth International Joint Conference on AI (pp.305-310). Cambridge, MA: MIT Press.

[3] Rumelhart, D.E., \& McClelland, J.L.(1986). Parallel distributed processing: exploration in the microstructure of cognition(Vols. 1 \& 2). Cambridge, MA : MIT Press.

[4] Duda, R.O., \& Hart, P.E.(1973). Pattern classification and scene analysis New York: John Wiley \& Sons.

[5] Werbos, P. (1975). Beyond regression: New tools for prediction and analysis in the behavioral sciences (Ph.D. dissertation). Harvard University.

[6] Parker, D.(1985). Learning logic (MIT Technical Report TR-47). MIT Center for Research in Computational Economics and Management Science.

[7] Kirkpatrick, S., Gelatt,Jr., C.D., and M.P. Vecchi 1983. Optimization by simulated annealing. Science 220(4598).

[8] Russel, S., \& Norvig, P. (1995). Artificial intelligence: A modern approach. Englewood Cliffs, NJ:Prentice-Hall.

[9] Mitchell, T.M., 1997.Machine Learning, New York: McGraw-Hill.

[10] Kirkpatrick, S., 1984 “Optimization by simulated annealing: Quantitative Studies,” Journal of Statistical Physics, vol.34,pp.975-986.

[11] Kennedy, J., Eberhart, R., 1995. "Particle swarm optimization”, IEEE International Conference on Neural Networks, Perth, WA , (pp.1942 - 1948), Australia.

[12] Engelbrecht, A.P., 2007, Computational Intelligence, England: John Wiley \& Sons. 
International Journal of Computer Science, Engineering and Applications (IJCSEA) Vol.1, No.6, December 2011

\section{Authors}

Sriram G. Sanjeevi has B.E. from Osmania University, M.Tech from M.I.T. Manipal, Mangalore University and $\mathrm{PhD}$ from IIT, Bombay. He is currently Associate Professor \& Head of Computer Science \& Engineering Dept., NIT Warangal. His research interests are Neural networks, Machine learning and Soft computing.

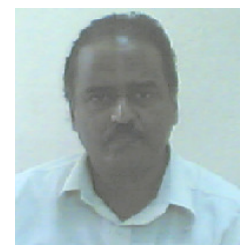

A. N. Nikhila has B.Tech(CSE) from NIT Warangal. Her research interests are Neural networks, Machine learning.

Thaseem Khan has B.Tech(CSE) from NIT Warangal. His research interests are Neural networks, Machine learning.

G. Sumathi has B.Tech(CSE) from NIT Warangal. Her research interests are Neural networks, Machine learning. 